\title{
A combination of TDM and KSAM to determine initial feasible solution of transportation problems
}

\author{
Muhammad Sam'an', Yahya Nur Ifriza ${ }^{2}$ \\ ${ }^{1}$ Postgraduate Student, Faculty of Technology Management and Business, Universiti Tun Hussein Onn Malaysia, \\ Malaysia \\ ${ }^{2}$ Department of computer science, Universitas Negeri Semarang, Indonesia
}

\begin{tabular}{l}
\hline Article Info \\
\hline Article history: \\
Received Jan 22, 2021 \\
Revised Feb 26, 2021 \\
Accepted March 15, 2021 \\
\hline
\end{tabular}

\section{Keywords:}

Transportation problem Initial feasible solution Approximation method Total difference method

\begin{abstract}
In case of the Transportation Problem (TP), it was found that TP had equal the smallest $c_{i j}$ so that the existing methods will be generated two or more IFS values. The newly developed algorithm is generated through a combination of Total Difference Method (TDM) and Karagul-Sahin Approximation Method (KSAM) algorithm, is capable to determine the initial feasible solution of TP. Based on the numerical illustration of the TP example to evaluate the performance of the new proposed algorithm. The computational performances have been compared to the existing methods (TDM1 and KSAM) and the results shown this algorithm achieved better performance than the existing methods for TP example. The recommendation of the research is the new proposed algorithm integration with Stepping Stone and MODI methods in future research to evaluate the optimal solution of TP and determination of optimal solution in the event of information uncertainty about the parameters of the TP.
\end{abstract}

This is an open-access article under the CC BY-SA license.

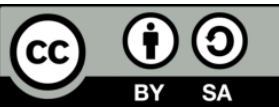

\section{Corresponding Author: \\ Muhammad Sam'an, \\ Postgraduate Student, \\ Faculty of Technology Management and Business, \\ Universiti Tun Hussein Onn Malaysia (UTHM), \\ Batu Pahat, Johor, 86400, Malaysia. \\ Email: muhammad.92sam@gmail.com}

\section{INTRODUCTION}

The method of finding Initial Feasible Solution (IFS) by assigning the smallest $c_{i j}$ as base cell in Transportation Problem (TP) table has been widely used by several algorithms such as classical algorithm namely Least Cost Method (LCM) takes into account the smallest $c_{i j}$ in the TP table and Vogel's Approximation Method (VAM) takes into account the highest penalty $c_{i j}$ which is denotes the difference between the smallest $c_{i j}$ and the next smallest $c_{i j}$ in each row and each column of the TP table.

Subsequently, various types of finding IFS methods by determining the smallest $c_{i j}$ of TP table were introduced by many researchers [1]-[3]. The pointer $c_{i j}$ calculated by selecting the difference from the highest $c_{i j}$ and second-highest $c_{i j}$ for each row and each column, unlike VAM [4]. Total Opportunity Cost Table (TOCT) calculate the Distribution Indicators (DI) by the difference of the greatest unit $c_{i j}$ and second gretest unit $c_{i j}$, then the highest two DI are selected as the base cell, and loads are imposed on the original TP corresponding to the base cells of the TOCT [5]. The lowest Allocation Method (LAM) was developed from LCM that also take into account the minimum of supply or demand for allocation in the smallest $c_{i j}$ of TP table 
[6]. Advanced Vogel's Approximation Method (AVAM) to overcome case of TP which has the equal smallest $c_{i j}$ in the TP table [7]. TOCM-SUM Approach is calculated by the pointer $c_{i j}$ for each row and column of the TOCM by selecting the sum of all entries in the respective row or column and made a maximum possible allocation to the smallest $c_{i j}$ cell corresponding to the highest pointer $c_{i j}$ [8]. Tuncay Cana's Approximation Method determined to the cell having the nearest $c_{i j}$ to this average $c_{i j}$ considering the demand and production constraints [9]. Incessant Allocation Method (IAM) [10] and Allocation Table Method (ATM) [11] are iterative methods based on the allocation table. Modification of TDM (Total Different Method) 1 considers penalty only for a row of TP table [12]. Global Minimum Method (GMM) [13]. Azad Hasan Method (AHM) is an effective algorithm for allocating the lowest number of demands and supplies to the lowest distribution cost of TP [14], [15]. The modification of TDM by adding the rules for selecting the highest penalty value and checking the lowest distribution cost followed by a combination of TOCM and TDM modification [16]. Total Opportunity Cost Matrix - Supreme Cell [17]. The novel approximation method is called Karagul-Sahin Approximation Method (KSAM) [18]. Zack algorithm [19] and Key Cell method (KCM) based avoiding maximum cost cells for making allocation [20]. An innovative strategy to obtain IBFS of TP based on penalty value [21]. The bottleneck transportation problem is based on pseudo cost to repair the IBFS that was obtained by classical transportation algorithm [22]. The revised ASM method [23]. The combination of TOCM and ASM (TOCM-ASM) [24], [25].

All proposed methods above by previous researchers illustrated the importance of taking the smallest $c_{i j}$ of TP as a base cell for allocating the numbers of supplies and demands of TP because it will affect the value of IFS. But, these proposed methods will only run perfectly if the TP does not have equal smallest $c_{i j}$ during the TP computations. Whenever there is equal smallest $c_{i j}$ in TP computations, the outcomes of the methods will fail to determine the smallest $c_{i j}$. Several methods have proposed alternatives solution by choosing one of the equal smallest $c_{i j}$ value available. Nevertheless, these alternative solutions for TP will result in more than one IFS value. Consequently, the IFS value generated will be different and it is depending on the smallest $c_{i j}$ value is chosen which is used to compute the IFS.

Therefore, the objective of this research is to overcome the limitations and complications of having two or more IFS values generated through the modification of TDM and followed by integrating with KSAM algorithm. This new algorithm is proposed which is design to obtain optimal IFS value of TP.

\section{MODELLING OF TRANSPORTATION PROBLEM}

The modeling of transportation problems (TP) can be formulated the following equation to determine the approximation value of $x_{i j}$ that minimizes the total distribution cost as follows,

subject to

$$
\operatorname{minT}=\sum_{i=1}^{m} \sum_{j=1}^{n} c_{i j} x_{i j}
$$

$$
\begin{aligned}
& \sum_{j=1}^{n} x_{i j} \leq s_{i} \\
& \sum_{i=1}^{m} x_{i j} \leq d_{j}
\end{aligned}
$$

and

$$
x_{i j} \geq 0 \forall i, j(i=1,2, \ldots, m ; j=1,2, \ldots, n) .
$$

where m represents a total of supply, n represents a total of demand, $s_{i}$ is $i^{\text {th }}$ suppply, $d_{j}$ is $j^{\text {th }}$ demand, $c_{i j}$ is distribution cost from $i^{\text {th }}$ suppply to $j^{\text {th }}$ demand, $x_{i j}$ is the number of approximation unit to assign from $i^{\text {th }}$ suppply to $j^{\text {th }}$ demand, minT is minimal total distribution cost.

If total supply identically with the total demand is called a balance TP and given as,

$$
\sum_{i=1}^{m} s_{i}=\sum_{j=1}^{n} d_{j}
$$

\section{THE NEW PROPOSED ALGORITHM}

The setting of the smallest $c i j$ which has equal values in the base cells in TP table affects the location of $x_{i j}$ allocated on the resulting IFS value depends on the setting of the smallest $c i j$. The new proposed algorithm is based on the modification cation of the TDM algorithm and is integrated with the KSAM algorithm, it is advantageous that the original of TDM and KSAM algorithms are shown as is Algorithm 1 in shown Figure 1 and Figure 2, respectively.

J. Soft. Comp. Explor., Vol. 2, No. 1, March 2021: 17 - 24

DOI: 


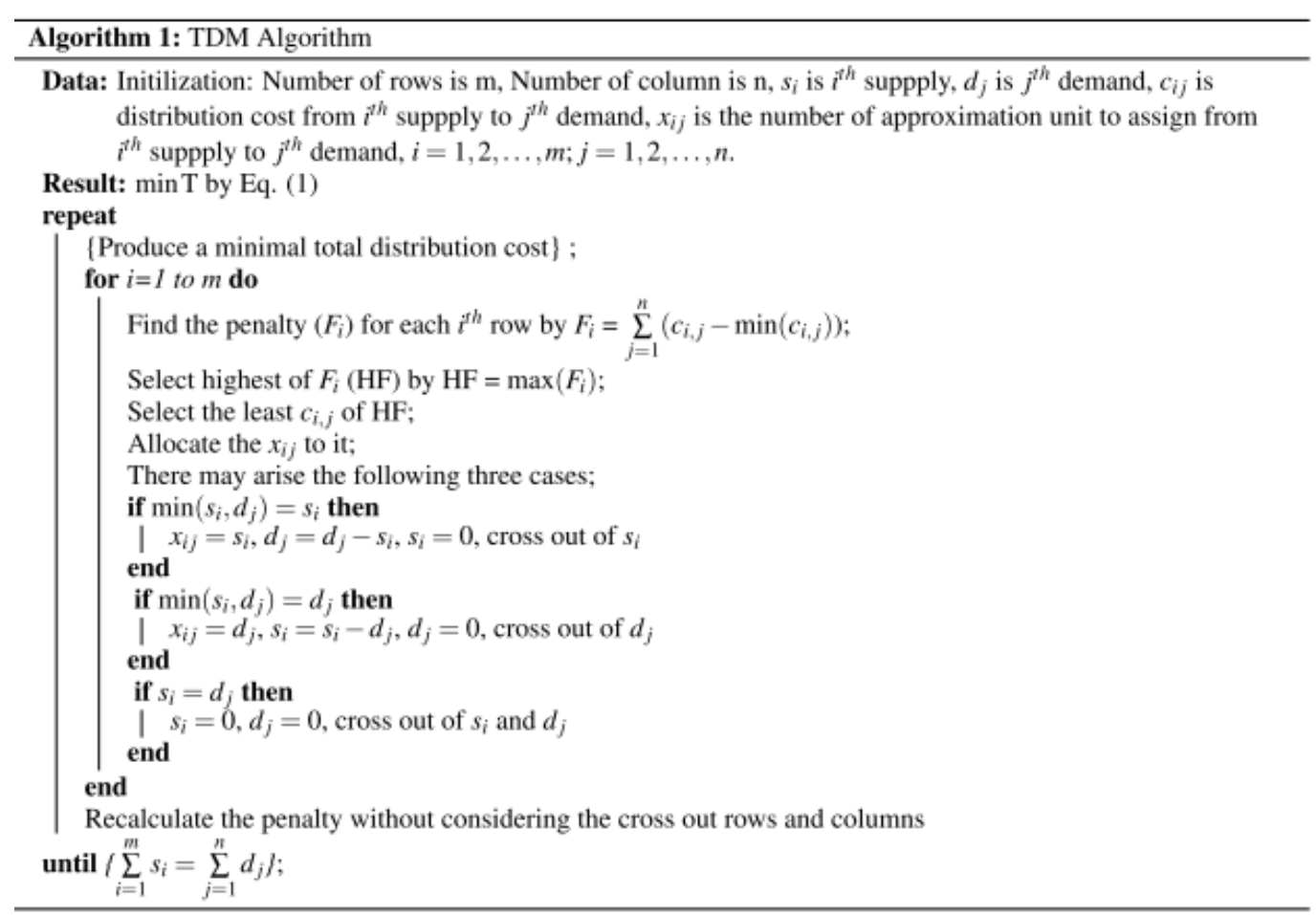

Figure 1. TDM1 algorithm

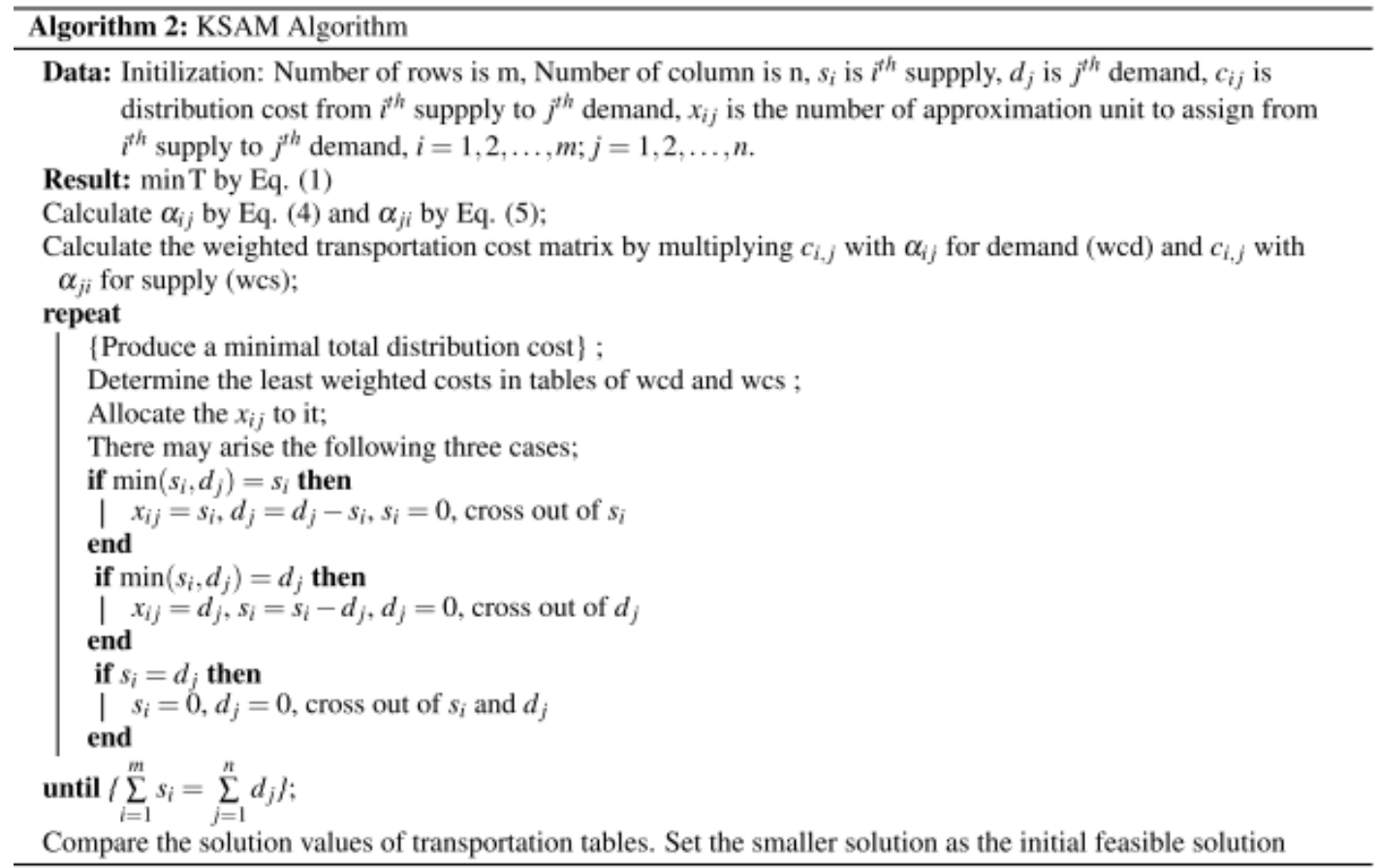

Figure 2. KSAM algorithm

The new proposed algorithm starts by checking either Eq. (5) is satisfied or not and followed by calculation of proportional demand matrix $\left(\alpha_{i j}\right)$ and proportional supply matrix $\left(\alpha_{j i}\right)$ by using Eq. (6) and (7), respectively [11].

$$
\begin{aligned}
& \alpha_{i j}=\frac{d_{j}}{s_{i}}, i=1,2, \ldots, m ; j=1,2, \ldots, n . \\
& \alpha_{j i}=\frac{s_{i}}{d_{j}}, j=1,2, \ldots, n ; i=1,2, \ldots, m .
\end{aligned}
$$




$$
\alpha_{i j} \alpha_{j i}=1
$$

Determine the sum of $\alpha_{i j}$ and $\alpha_{j i}$, the resulting value is called weighted distribution cost matrix $\left(\omega_{i j}\right)$. The new proposed algorithm in detail is Algorithm 3 in shown Figure 3.

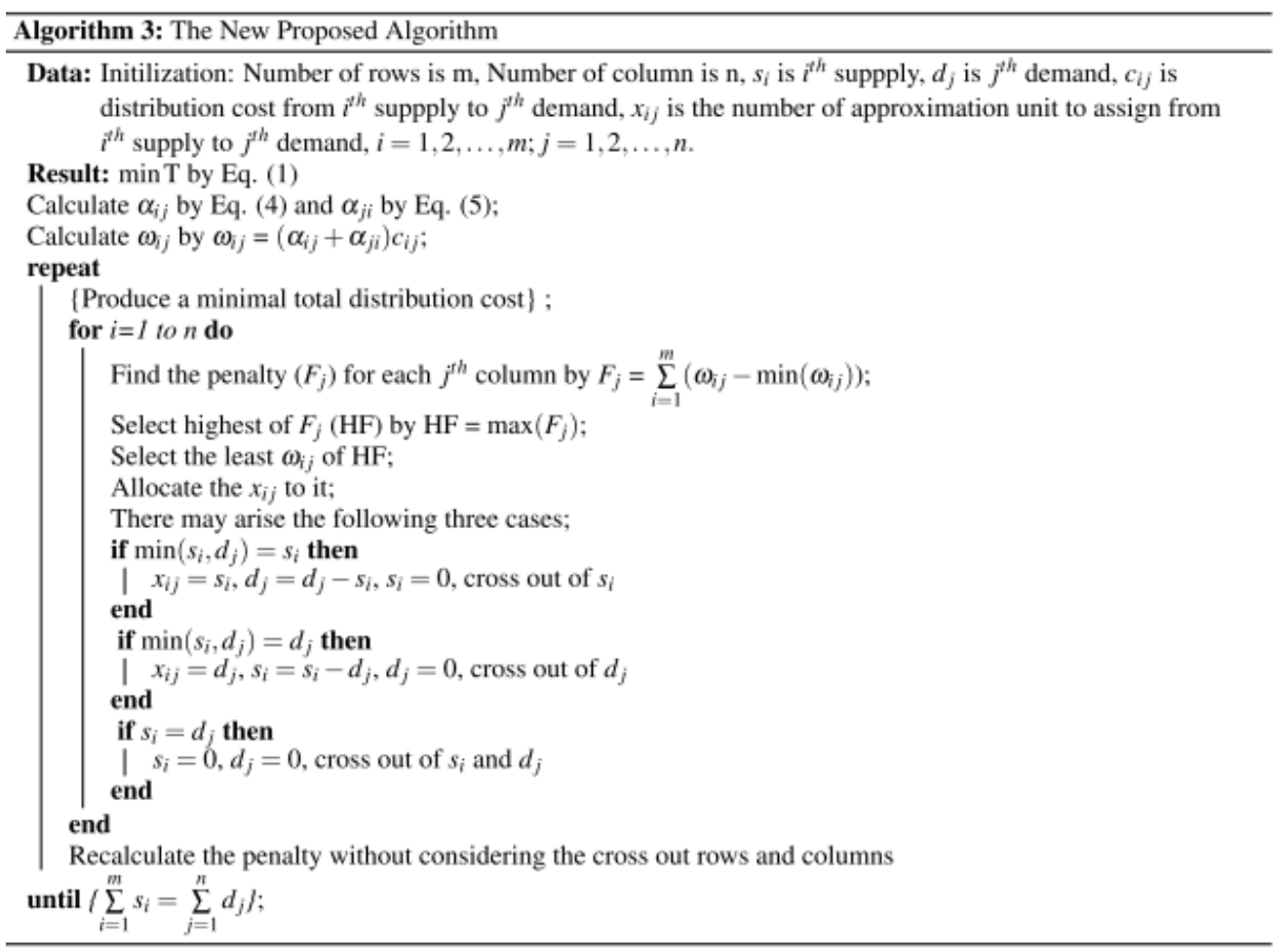

Figure 3. The new proposed algorithm

\section{NUMERICAL ILLUSTRATIONS}

The numerical illustration used an example of a transportation problem to explain the new proposed algorithm is shown Table 1 ([16]).

Table 1. An original data of transportation problem in example.

\begin{tabular}{|c|c|c|c|c|c|c|c|}
\hline \multirow{2}{*}{ Sources } & \multicolumn{6}{|c|}{ Destination } & \multirow[t]{2}{*}{$s_{i}$} \\
\hline & $\mathrm{D}_{1}$ & $\mathrm{D}_{2}$ & $\mathrm{D}_{3}$ & $\mathrm{D}_{4}$ & $D_{5}$ & $\mathrm{D}_{6}$ & \\
\hline $\mathrm{S}_{1}$ & 9 & 12 & 9 & 6 & 9 & 10 & 2 \\
\hline $\mathrm{S}_{2}$ & 7 & 3 & 7 & 7 & 5 & 5 & 5 \\
\hline $\mathrm{S}_{3}$ & 6 & 5 & 9 & 11 & 3 & 11 & 6 \\
\hline $\mathrm{S}_{4}$ & 6 & 8 & 11 & 2 & 2 & 10 & 9 \\
\hline$d_{j}$ & 2 & 2 & 2 & 4 & 4 & 6 & \\
\hline
\end{tabular}

The solution of the transportation problem example 1 in Table 1 is solved by using Algorithm 3 as follows:

Step 1 : Calculating $\alpha_{i j}$ and $\alpha_{j i}$. For $i=1,2,3,4 ; j=1,2,3,4,5,6$ are obtained as bellows

$$
\alpha_{46}=\left(\begin{array}{llllll}
1 & 1 & 2 & 2 & 2 & 3 \\
0.4 & 0.4 & 0.8 & 0.8 & 0.8 & 1.2 \\
0.33 & 0.33 & 0.67 & 0.67 & 0.67 & 1 \\
0.22 & 0.22 & 0.44 & 0.44 & 0.44 & 0.67
\end{array}\right) \text { and } \alpha_{64}=\left(\begin{array}{llllll}
1 & 1 & 0.5 & 0.5 & 0.5 & 0.33 \\
2.5 & 2.5 & 1.25 & 1.25 & 1.25 & 0.83 \\
3 & 3 & 1.5 & 1.5 & 1.5 & 1 \\
4.5 & 4.5 & 2.25 & 2.25 & 2.25 & 1.5
\end{array}\right)^{T}
$$


Step 2: Calculating $\omega_{i j}$. For $i=1,2,3,4 ; j=1,2,3,4,5,6$ is produced as bellow

$\boldsymbol{\omega}_{46}=\left(\begin{array}{llllll}18 & 24 & 22.5 & 15 & 22.5 & 33.3 \\ 23.2 & 8.7 & 14.35 & 14.35 & 10.25 & 10.17 \\ 20 & 16.67 & 19.5 & 23.8 & 6.5 & 22 \\ 28.33 & 37.78 & 29.69 & 5.39 & 5.39 & 21.67\end{array}\right)$

Step 3: Calculating $F_{j}$. For $j=1$ so $F_{1}$ is obtained as bellows

$$
\begin{aligned}
& F_{1} \text { of column-1 }=14.63 \\
& F_{1} \text { of column-2 }=52.34 \\
& F_{1} \text { of column-3 }=28.59 \\
& F_{1} \text { of column- } 4=37.01 \\
& F_{1} \text { of column-5 }=23.03 \\
& F_{1} \text { of column-6 }=46.5
\end{aligned}
$$

Step 4: Selecting the highest of $F_{1}$ is $F_{1}$ of column-2

Step 5: Selecting the least $\omega_{i j}$ in $F_{1}$ of column-2 is $\omega_{22}$.

Step 6: Allocating the $x_{22}$ to $\omega_{22}$ with $x_{32}=\min \left(s_{2}, d_{2}\right)=\min (2,5)=2$ such that $s_{2}=s_{2}-d_{2}=5-2=$ 3 and $d_{2}=d_{2}-d_{2}=2-2=0$. Since $s_{2} \neq 0$ and $d_{2}=0$ then cross out of $d_{2}$.

Step 7: Re-calculating the penalty without considering $d_{2}$ such that for $j=2$ is obtained as bellows.

$$
\begin{aligned}
& F_{2} \text { of column-1 }=14.63 \\
& F_{2} \text { of column-3 }=28.59 \\
& F_{2} \text { of column-4 }=37.01 \\
& F_{2} \text { of column-5 }=23.03 \\
& F_{2} \text { of column-6 }=46.5
\end{aligned}
$$

\begin{tabular}{|c|c|c|c|c|c|c|c|}
\hline \multirow{2}{*}{ Sources } & \multicolumn{6}{|c|}{ Destination } & \multirow[t]{2}{*}{$s_{i}$} \\
\hline & $\mathrm{D}_{1}$ & $\mathrm{D}_{2}$ & $\mathrm{D}_{3}$ & $\mathrm{D}_{4}$ & $\mathrm{D}_{5}$ & $\mathrm{D}_{6}$ & \\
\hline $\mathrm{S}_{1}$ & 0 & 0 & 0 & 0 & 0 & 2 & 2 \\
\hline $\mathrm{S}_{2}$ & 0 & 2 & 0 & 0 & 0 & 3 & 5 \\
\hline $\mathrm{S}_{3}$ & 2 & 0 & 4 & 0 & 0 & 0 & 6 \\
\hline $\mathrm{S}_{4}$ & 0 & 0 & 0 & 4 & 4 & 1 & 9 \\
\hline$d_{j}$ & 2 & 2 & 2 & 4 & 4 & 6 & \\
\hline
\end{tabular}

Step 8: Repeat Step 3 until Step 6 such that $\sum_{i=1}^{m} s_{i}=\sum_{j=1}^{n} d_{j}$. The final result is shown in Table 2 .

Table 2. The new proposed algorithm with a penalty.

\begin{tabular}{cllllll}
\hline \multirow{2}{*}{ Sources } & \multicolumn{7}{c}{ Destination } \\
\cline { 2 - 7 } & $\mathrm{D}_{1}$ & $\mathrm{D}_{2}$ & $\mathrm{D}_{3}$ & \multicolumn{1}{c}{$\mathrm{D}_{4}$} & $\mathrm{D}_{5}$ & $\mathrm{D}_{6}$ \\
\hline $\mathrm{S}_{1}$ & 18 & 24 & 22.5 & 15 & 22.5 & 33.3 \\
$\mathrm{~S}_{2}$ & 23.2 & 8.7 & 14.35 & 14.35 & 10.25 & 10.17 \\
$\mathrm{~S}_{3}$ & 20 & 16.67 & 19.5 & 23.8 & 6.5 & 22 \\
$\mathrm{~S}_{4}$ & 28.33 & 37.78 & 29.69 & 5.39 & 5.39 & 21.67 \\
\hline$F_{1}$ & 14.63 & 52.34 & 28.59 & 37.01 & 23.08 & 46.5 \\
$F_{2}$ & 14.63 & 0 & 28.59 & 37.01 & 23.08 & 46.5 \\
$F_{3}$ & 12.33 & 0 & 13.13 & 28.05 & 18.22 & 12 \\
$F_{4}$ & 12.33 & 0 & 13.13 & 0 & 18.22 & 12 \\
$F_{5}$ & 12.33 & 0 & 13.13 & 0 & 0 & 12 \\
$F_{6}$ & 2 & 0 & 3 & 0 & 0 & 11.33 \\
$F_{7}$ & 2 & 0 & 3 & 0 & 0 & 0 \\
$F_{8}$ & 2 & 0 & 0 & 0 & 0 & 0 \\
$F_{9}$ & 0 & 0 & 0 & 0 & 0 & 0 \\
\hline
\end{tabular}

Step 9: Finally, calculating minimal total distribution cost by using Eq. (1). The result of minT is 115 . The feasible solution table of example 1 can be seen Table 3.

Table 3. The feasible solution table of example. 


\section{DISCUSSION}

The application of the new proposed has been described with a numerical illustration of transportation problem example which has not only one or more the smallest $c_{i j}$ of the TP table. The solving of TP example used the Algorithm 3 did not raise the potential to produce more than one IFS. It can be shown from the highest penalty used to determine which is base cell has not the equal value.

Meanwhile, the solving of TP example used Algorithm 3 (TDM 1[12]) raised the potential to produce more than on IFS. It is because the highest penalty has equal value. The highest penalty of TP example was produced by Algorithm 1 is shown in Table 4. If we compare the highest penalty obtained by Algorithm 1 with Algorithm 3, then Algorithm 3 is better at producing the highest penalty than Algorithm 1. Besides that, Algorithm 1 does not provide additional conditions if there is the highest penalty with the equal value such that it can potentially be Algorithm 1 cannot be made to solve TP example.

Table 4. The penalties of transportation problem in example generated TDM 1.

\begin{tabular}{clllllll}
\hline Sources & \multicolumn{9}{c}{ Destination } & $F_{i}$ \\
\cline { 2 - 7 } & $\mathrm{D}_{1}$ & $\mathrm{D}_{2}$ & $\mathrm{D}_{3}$ & $\mathrm{D}_{4}$ & $\mathrm{D}_{5}$ & $\mathrm{D}_{6}$ & \\
\hline $\mathrm{S}_{1}$ & 9 & 12 & 9 & 6 & 9 & 10 & 19 \\
$\mathrm{~S}_{2}$ & 7 & 3 & 7 & 7 & 5 & 5 & 16 \\
$\mathrm{~S}_{3}$ & 6 & 5 & 9 & 11 & 3 & 11 & 27 \\
$\mathrm{~S}_{4}$ & 6 & 8 & 11 & 2 & 2 & 10 & 27 \\
\hline
\end{tabular}

Furthermore, the solving of TP example used Algorithm 2 (KSAM [18]) showed also the potential to generate not only one or more on IFS. It is caused by weighted transportation cost matrix by demand (WCD) or weighted transportation cost matrix by supply (WCS) which is assigned to select the smallest $c_{i j}$ as a base cell has equal value. The value of WCD and WCS on TP example as follows,

$$
\begin{aligned}
\mathrm{WCD} & =\left(\begin{array}{llllll}
9 & 12 & 18 & 12 & 18 & 30 \\
2.8 & 1.2 & 5.6 & 5.6 & 4 & 6 \\
2 & 1.67 & 6 & 7.3 & 2 & 11 \\
1.3 & 1.78 & 4.89 & 0.89 & 0.89 & 6.67
\end{array}\right) \text { and } \\
\mathrm{WCS} & =\left(\begin{array}{llllll}
9 & 12 & 4.5 & 3 & 4.5 & 3.3 \\
17.5 & 7.5 & 8.75 & 8.75 & 6.25 & 4.17 \\
18 & 15 & 13.5 & 16.5 & 4.5 & 11 \\
27 & 36 & 24.75 & 4.5 & 4.5 & 15
\end{array}\right)^{T}
\end{aligned}
$$

If we also compare WCS and WCD obtained by Algorithm 2 with the penalty of Algorithm 3, then Algorithm 3 is better than Algorithm 2. Similarly, with the TDM 1, Algorithm 2 does not provide additional conditions if there is the WCD or WCS with the equal value such that it can potentially be Algorithm 2 cannot be made to solve TP example. TDM 1 and KSAM add criteria by freely selected the equal highest penalty (Algorithm 1) and equal value of WCD or WCS (Algorithm 2) so that IFS will be obtained. Therefore, the result comparison Of IFS between the new proposed method (Algorithm 3) with the existing methods (Algorithm 1 [12] and Algorithm 2 [18]) is shown Table 5. In this table can be seen the new proposed algorithm solved the TP example better than TDM 1 and KSAM. The algorithm of TDM and KSAM produced two IFS. The first IFS produced by TDM 1 algorithm is better than the new proposed algorithm and otherwise, the second IFS produced by TDM algorithm fewer results compared to the new proposed algorithm. Meanwhile, both IFS (WCS) generated by KSAM algorithm is better than the new proposed algorithm.

Table 5. The result comparison of IFS.

\begin{tabular}{ll}
\hline The existing method & Initial Feasible Solution (IFS) \\
\hline \multirow{2}{*}{ TDM1 } & $117(1)$ \\
& $110(2)$ \\
& $123(\mathrm{WCD})$ \\
& $121(\mathrm{WCS}-1)$ \\
KSAM & $131(\mathrm{WCS}-2)$ \\
& 115 \\
\hline
\end{tabular}




\section{CONCLUSION}

The importance of setting the smallest $c_{i j}$ as the base cell for finding IFS of TP to determine the optimal solution which is the minimum total cost. The new proposed algorithm is developed based on the KSAM characteristics improvisation plus integration of modified TDM algorithm. It also considers the supply and demand coverage ratio (weights) as well as the smallest $c_{i j}$ and included the penalties calculations for each column. This new proposed algorithm is capable to find the IFS effectively and efficiently. The numerical illustration of TP example indicated that the new proposed algorithm numerical results is comparable to the existing methods. The new proposed algorithm achieved better performance than TDM 1 and KSAM algorithms for TP example. In addition, it is also recommended that the new proposed algorithm integration with Stepping Stone and MODI methods in future research to evaluate the optimal solution of TP and determination of optimal solution in the event of information uncertainty about the parameters of TP.

\section{REFERENCES}

[1] D. Aprilianto, "SVM optimization with correlation feature selection based binary particle swarm optimization for diagnosis of chronic kidney disease," J. Soft Comput. Explor., vol. 1, no. 1, pp. 24 $31,2020$.

[2] R. H. Saputra and B. Prasetyo, "Improve the accuracy of c4.5 algorithm using particle swarm optimization ( pso ) feature selection and bagging technique in breast cancer diagnosis," J. Soft Comput. Explor., vol. 1, no. 1, pp. 47-55, 2020.

[3] I. E.Tiffani, "Optimization of naïve bayes classifier by implemented unigram, bigram, trigram for sentiment analysis of hotel review," J. Soft Comput. Explor., vol. 1, no. 1, pp. 1-7, 2020.

[4] A. Khan, "A Re-solution of the Transportation Problem: An Algorithmic Approach," Jahangirnagar Univ. J. Sci., vol. 34, pp. 49-62, 2011.

[5] M. A. Islam, M. M. Haque, and M. S. Uddin, "Extremum difference formula on total opportunity cost: a transportation cost minimization technique," Prime Univ. Journa, vol. 6, no. 2, pp. 125-130, 2012.

[6] M. Ashraful Babu, M. Abu Helal, M. S. Hasan, and U. K. Das, "Lowest Allocation Method (LAM): a new approach to obtain feasible solution of transporta-tion model," Int. J. Sci. Eng. Res., vol. 4, no. 11, 2013.

[7] U. Kanti Das, M. Ashraful Babu, A. Rahman Khan, and D. Sharif Uddin, “Advanced Vogel's Approximation Method (AVAM): a new approach to determine penalty cost for better feasible solution of transportation problem," Int. J. Eng. Res. Technol., vol. 3, p. 182, 2014.

[8] A. R. Khan, V. Adrian, N. Sultana, and S. S. Ahmed, "Determination of initial basic feasible solution of a transportation problem: a TOCM-SUM approach," Bul. Institutului Politeh. Din Iasi, vol. 61, pp. $39-49,2015$.

[9] T. Can, "Tuncay Can's Approximation method to obtain initial basic feasible solution to transport problem," Appl. Comput. Math., vol. 5, no. 2, p. 78, 2016, doi: 10.11648/j.acm.20160502.17.

[10] M. M. Ahmed, A. R. Khan, F. Ahmed, and M. S. Uddin, "Incessant Allocation Method for Solving Transportation Problems," Am. J. Oper. Res., vol. 06, no. 03, pp. 236-244, 2016, doi: 10.4236/ajor.2016.63024.

[11] M. M. Ahmed, A. R. Khan, M. S. Uddin, and F. Ahmed, "A new approach to solve transportation problems," Open J. Optim., vol. 05, no. 01, pp. 22-30, 2016, doi: 10.4236/ojop.2016.51003.

[12] E. Hosseini, "Three new methods to find initial basic feasible solution of transportation problems," Appl. Math. Sci., vol. 11, pp. 1803-1814, 2017, doi: 10.12988/ams.2017.75178.

[13] Y. Harrath and J. Kaabi, "New heuristic to generate an initial basic feasible solution for the balanced transportation problem," Int. J. Ind. Syst. Eng., vol. 30, no. 2, p. 193, 2018, doi: 10.1504/ijise.2018.10016216.

[14] S. M. Abul Kalam Azad and M. Kamrul Hasan, "An effective algorithm to solve cost minimising transportation problem," Int. J. Math. Oper. Res., vol. 15, no. 4, pp. 434-445, 2019, doi: 10.1504/IJMOR.2019.103005.

[15] Z. H. Radthy, F. H. Maghool, and A. H. Khaleel, "Application the linear programming according to transportation problem on real data," Int. J. Sci. Technol. Res., vol. 8, no. 1, pp. 100-102, 2019.

[16] B. Amaliah, C. Fatichah, and E. Suryani, "Total opportunity cost matrix - Minimal total: A new approach to determine initial basic feasible solution of a transportation problem," Egypt. Informatics J., vol. 20, no. 2, pp. 131-141, 2019, doi: 10.1016/j.eij.2019.01.002.

[17] B. Amaliah, C. Fatichah, E. Suryani, and A. Muswar, "Total opportunity cost matrix - Supreme cell: A new method to obtain initial basic feasible solution of transportation problems," in ACM International Conference Proceeding Series, 2020, pp. 151-156, doi: 10.1145/3411174.3411198.

[18] K. Karagul and Y. Sahin, "A novel approximation method to obtain initial basic feasible solution of transportation problem," J. King Saud Univ. - Eng. Sci., vol. 32, no. 3, pp. 211-218, 2020, doi: 10.1016/j.jksues.2019.03.003. 
[19] Z. U. Rizqi, "Zack algorithm: A heuristic approach to solve transportation problem," in Proceedings of the International Conference on Industrial Engineering and Operations Management, 2019, pp. $1127-1131$

[20] L. Kaur, M. Rakshit, and S. Singh, "An alternate approach for finding the initial basic feasible solution of transportation problem," Int. J. Sci. Technol. Res., vol. 8, no. 9, pp. 442-447, 2019.

[21] P. Sumathi and C. V. Sathiya Bama, "An innovative route to acquire least cost in transportation problems," Int. J. Eng. Adv. Technol., vol. 9, no. 1, pp. 5368-5369, 2019, doi: 10.35940/ijeat.A3070.109119.

[22] B. Mallia, M. Das, and C. Das, "A New Algorithm for Bottleneck Transportation Problem," Int. J. Innov. Technol. Explor. Eng., vol. 8, no. 8, pp. 419-423, 2019.

[23] R. Murugesan and T. Esakkiammal, "Determination of best initial basic feasible solution of a transportation problem: A TOCM-ASM approach," Adv. Math. Sci. J., vol. 9, no. 7, pp. 4563-4577, 2020, doi: 10.37418/amsj.9.7.25.

[24] R. Murugesan and T. Esakkiammal, "An improved ASM method for the transportation problem," Adv. Math. Sci. J., vol. 9, no. 10, pp. 8259-8271, 2020, doi: 10.37418/amsj.9.10.55.

[25] R. Murugesan and T. Esakkiammal, "TOCM-VAM method versus asm method in transportation problems,” Adv. Math. Sci. J., vol. 9, no. 6, pp. 3549-3566, 2020, doi: 10.37418/amsj.9.6.34.

J. Soft. Comp. Explor., Vol. 2, No. 1, March 2021: 17 - 24

DOI: 